\title{
Anti-caries Potential of Silver Nanoparticles via Modulation of Free Calcium Activity within the Plaque Fluid of the Oral Biofilm: A Pilot Study
}

Craig Callister, Matthew Callister ${ }^{*}$, Michael Nolan and Ryan Nolan

Department of Dental Research, Novus Research, Dental Science, UT 84651, USA

*Corresponding author: Matthew Callister, Section of Dental Research, Novus Research, United States, 1172 E. 100 N. St. \#6 Payson, UT 84651 , USA, Tel: 1-801-465-3691; E-mail: mcallister@elementasilver.com

Received date: December 31, 2018; Accepted date: January 07, 2019; Published date: January 10, 2019

Copyright: ( 2018 Callister C, et al. This is an open-access article distributed under the terms of the Creative Commons Attribution License, which permits unrestricted use, distribution, and reproduction in any medium, provided the original author and source are credited.

\begin{abstract}
Background: The specific aim of this study was to evaluate the effects of silver nanoparticles (AgNPs) on calcium ion activity within the plaque fluid of oral biofilms.

Materials and Methods: An in ex-vivo prospective, randomized, controlled, double-blind study model was used. Two case study tests-one on a plaque from nine caries-free subjects (averaged samples) and another on a plaque from eight caries-free subjects (pooled samples) were done. All subjects abstained from brushing for 48 hours prior to the collection of plaque. All subjects were randomly assigned to a rinse which either contained calcium chloride $(0.2 \%$ control), or calcium chloride $(0.2 \%)$ and AgNPs $(10 \mathrm{ppm})$. Plaque samples were taken prior to and 60 minutes after rinsing and spun down using a centrifuge and analyzed using calcium selective electrodes to determine calcium activity in plaque extra orally. The effects were analyzed using a two-sided T-test to implore significance.
\end{abstract}

Results: Test and control mouthwashes produced differing levels of free calcium in the plaque. The test rinse significantly increased the calcium ion activity in plaque by a significant degree $(p<0.05)$. The results were more significant for the pooled testing.

Conclusion: AgNPs show promise for being used in hygiene products for increasing calcium delivery into biofilms, offering the opportunity to increase remineralization and reduce acidity from oral biofilms via calcium entry.

Keywords: Bioavailable calcium; Plaque fluid; Oral biofilm; Hydroxyapatite; Point of zero charge; Cation exchange capacity; Remineralization; Silver nanoparticles; $\mathrm{pH}$

\section{Introduction}

In the United States alone, Oral Biofilms (OB) cause billions of dollars of damage in the form of decay, periodontitis and tooth loss every year. The $\mathrm{OB}$ is a complex community of bacteria which accumulate on dental surfaces in the form of plaque or calculus. Over time the OB can undergo "shifts" which can lead to positive or negative outcomes based on the bacterial species present [1-3]. When disease processes take hold it can be observed that more opportunistic bacteria will change the conditions of the $\mathrm{OB}$ to suit their needs. In the case of anaerobic bacteria, this could be an environment more devoid of oxygen. In the case of acidogenic bacteria, this could be an environment that favors acid production in the presence of digestible carbohydrates and simple sugars $[2,4]$. Due to the fact that the $\mathrm{OB}$ is a community of different bacterial species, it has a complex wealth of genetic information sharing, and the ability to prevent changes to its community through mechanisms which are not all well-known. Due to this fact, many chemicals, and antibiotic driven approaches alone have failed to cause significant long term impacts on OB development and the resulting disease processes which ensue [5].

The release of acid from the bacterial $\mathrm{OB}$ after digestion of carbohydrates and simple sugars causes demineralization and subsequent caries [4]. Ultimately, unless the conditions within the OB are changed to encourage calcium entry and deposition, there are limitations to what the body can do to combat a well-established acidogenic OB. As can be seen from previous work, the OB exhibits a great deal of control over its internal ionic environment which directly contacts tooth structures $[4,6]$. As a result, when the $O B$ is not mechanically removed it undergoes bacterial shifts [1,3]. Depending on diet, hygiene, immune factors, and salivary quality and quantity, these shifts can lead to favor or oppose more opportunistic bacteria involved in the caries process $[1,3]$.

The most important building block for remineralization of enamel and dentinal surfaces is bioavailable calcium. Free calcium, which is exchangeable with mineral surfaces is referred to as "bioavailable calcium."

Under neutral to moderately basic conditions, bioavailable calcium can favorably react with and re-mineralize tooth structures via adsorption onto and absorption into the Hydroxyapatite (HA) crystal lattices [7]. The attraction of cations (specifically calcium) to enamel surfaces is dictated by many factors, but can be summed by Coulomb's force attraction equation with Calcium $\left(\mathrm{Ca}^{2+}\right)$ and $\mathrm{HA}(\mathrm{Eq}(1))$ below [8]:

$$
F(\text { attraction })=\frac{{ }_{C a}(2+)^{Z}{ }_{H A}(-)}{\varepsilon\left(R_{h}^{2}\right)}
$$




\section{$\mathrm{pH}<5.5$ Demineralization}

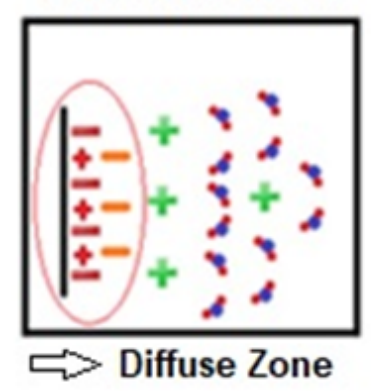

$\mathrm{pH}>7.0$ Remineralization

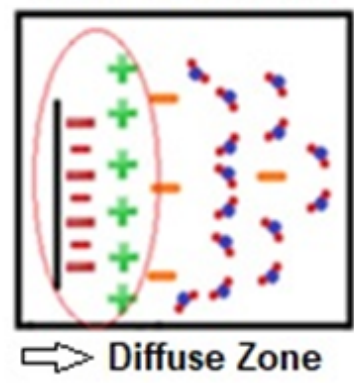

\section{HA Surface/Stern Layer}

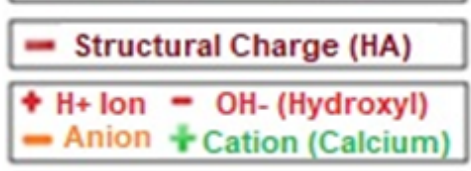

$\checkmark$ Water Hydration Sheath

Figure 1(a): Depiction of the $\mathrm{pH}$ dependency of HA surface charge via a stern layer model. This model emphasizes cation surface attraction is $\mathrm{pH}$ dependent, as increases in $\mathrm{pH}$ yield more negative HA surfaces. This increase in negative surface charge attracts more calcium ions, leading to remineralization.

As can be noted by Coulombs law (Equation 1-1) [8], when $\mathrm{pH}$ goes above the Point of Zero Charge (PZC) the force equation directly favors the attraction of positively charged calcium ions onto enamel HA surfaces which are more negatively charged due to saturation with hydroxyl anions (Figure 1(a)) $[9,10]$. As the $\mathrm{pH}$ of the plaque fluid increases, the net charge of HA becomes more negative. Most sources agree that in the presence of sodium chloride $>0.01 \mathrm{M}$, the PZC of HA is 7.6. However, it can be noted that the PCZ of enamel can be lower and varies. Therefore, above a $\mathrm{pH}$ of 7.0, and ideally in a range of 7.4-7.6 highly favours calcium attraction, leading to calcium ion adsorption and absorption with subsequent remineralization $[9,10]$. Plaque fluid is frequently cited as having above $0.01 \mathrm{M} \mathrm{NaCl}$, making this $\mathrm{pH}$ range an excellent approximation and agrees with clinical findings $[6,10]$. Due to plaque fluids constant contact with tooth structure and its impact on enamel dissolution, it is critical to modulate the calcium and subsequent $\mathrm{pH}$ in this fluid for remineralization to occur $[9,11]$. As the $\mathrm{pH}$ of the contacting solution increases, the surface charge of HA becomes more net negative from hydroxyl anion saturation. This enhances the remineralization cycle via adsorption and absorption of calcium. This model can be explained using the guoy-chapman modified stern model which dictates how charge based mineral surfaces can attract anions or cations based off of the combination of structural and $\mathrm{pH}$ based charge influences $[9,10]$. It can be noted that increasing $\mathrm{pH}$ levels and bioavailable calcium ions in plaque fluid is critical to the prevention of oral disease pathways [11].

Therefore, being able to increase the amount of bioavailable calcium in the $\mathrm{OB}$ is greatly valued due to its remineralization potential.

Although the lattice backbone of HA crystals is primarily composed of phosphate, an active portion of the mineral is the external charge fixed calcium layer, which in the extension of the gouy-chapman ionic mineral model is called the "stern layer"[9]. The externally positively charged stern layer serves as an interactive platform and reservoir for this type of mineral exchange via adsorption $[9,12,13]$. Bioavailable "free" calcium is also valued for its effect as a buffer, as well as ensuring the DS (Degree of saturation) is maintained from an external source instead of from dissolution of tooth structure $[6,11]$. Ultimately without bio-available calcium, remineralization is a chemical impossibility $[4,7,12]$.

It should be noted that previous work points out the value of bioavailable calcium and its effects on Biofilm cariogenicity. Margolis and Moreno, notes that bound calcium can account for up to $50 \%-75 \%$ of the calcium present in plaque [6]. Although this calcium has integrated into plaque and can no longer participate in direct reactions with tooth structures, it can still be considered a reservoir for calcium during acid attacks. It is possible to suggest that bound calcium deposits occur more readily in areas where the diffuse layer has become dehydrated or "collapsed", due to lack of oral saliva, or from localized periods of dry mouth. It could also be suggested that prevention of this collapse could induce a larger portion of the stern layer surrounding enamel surfaces to contain higher proportions of free calcium, but this remains theoretical in nature [12-14]. Because of this bound calcium it does not serve as a primary deterrent for prevention of acid attacks in the $\mathrm{OB}$ [4] but can be a reservoir for future release of calcium during acid attacks, when the $\mathrm{pH}$ of the biofilm is lowered. Should there be an increase in free calcium where acid attacks are prevalent, cariogenicity could be more easily controlled and biofeedback could be induced for bacterial species to promote the release of calcium ions [15]. This release could induce higher $\mathrm{pH} / \mathrm{Ca}^{2+}$ environments (where $\mathrm{Ca}^{2+}$ indicates free ion calcium activity $\left[\mathrm{Ca}^{2+}\right]$ ) [4]. For this reason, agents which can be used to deliver more free calcium directly to the $\mathrm{OB}$ would be of great scientific interest for potential caries prevention.

When an enamel crystal is depleted of its external charged stern layer and experiences too much damage to its phosphate backbone, remineralization attempts become more difficult [16,17]. This is especially true in cases of infected dentin-where remineralization cannot occur due to the loss of phosphate backbone, HA crystals, and collagen networks $[16,17]$. This can be due to ion competition and substitution-usually with carbonate ions, $\mathrm{pH}$-based lattice charge repulsion, and the limited bioavailability of various minerals in saliva also affects remineralization $[13,16]$. Remineralization approaches that have tried to incorporate calcium and phosphate together have the challenge of the low solubility of calcium phosphate salts, and interference from fluoride ions-especially in aqueous applications. This is likely due to the fact that phosphate groups have a high affinity for calcium ions in solution, thus preventing full dissociation and solubility [11]. This can limit the potential for use of calcium phosphates in aqueous dentrifices, which may precipitate prior to reaching tooth structures. However, incorporation of calcium salts can still be used as viable alternatives to maintain a degree of saturation in dental plaque for the purposes of remineralization, as well as buffering agents [18]. As the solubility equilibria approach a higher $\mathrm{pH}$, dependence on phosphate is lowered to maintain a degree of saturation due to excess calcium and hydroxyl groups [11].

Many researchers have theorized the composition of the $\mathrm{OB}$ and how it controls its internal ionic environment. As has been previously 
established in the literature, the OB contains many theoretical microchannels which control the internal and external ionic exchange between the two liquid mediums: plaque fluid and saliva $[4,6]$. Due to plaque fluid having a much higher flux in $\mathrm{pH}$ than saliva, neutralization of plaque fluid is critical in deterring extended periods of acidic demineralization [11]. The composition and structure of the $\mathrm{OB}$, as well as the chemical nature of saliva and plaque fluid, play large roles in the caries process $[4,11]$. One of the largest factors involved in the remineralization process of enamel surfaces involves having free calcium available for the enamel to utilize as building blocks for demineralized areas $[4,18]$. Although more research needs to be done, the cariogenic $\mathrm{OB}$ has developed methods to deter external ions such as calcium from freely entering and exchanging into the plaque fluid of the $\mathrm{OB}$, which directly contacts the enamel surfaces $[4,19]$. Cariogenic species have also been noted to be more tolerant to release of calcium ions, especially in low $\mathrm{pH}$ environments [19]. Due to the difficult nature of free ion diffusion into the plaque fluid of the $\mathrm{OB}$, alternative methods of improving this gradient will be discussed.

\section{The challenge of bioavailability: Ion gradients}

Ion gradients in the Oral cavity are complex and present multiple barriers of entry into the $\mathrm{OB}$ for ions and dissolved minerals. As can be illustrated in Figure 1(b), the ion gradients which control the diffusion of calcium, and other species of interest into the OB are as follows:

1) Salivary bioavailability and diffusion into Exopolysaccharide Slime Layer (EPS Matrix)

\section{2) Internal biofilm and plaque fluid}

3) Salivary pellicle, and Enamel surfaces (bacterial attachment, and ion competition for enamel surfaces)

These ion gradients can prevent ions of interest, such as calcium from being bioavailable to exchange at mineral surfaces where demineralization has occurred. Thus, improving the equilibrium and competition of desired mineral species past these gradients can greatly decrease the rate of potential demineralization and undesirable substitution occurring at enamel surfaces.

Saliva is the first gradient which soluble species must enter. It should be noted that depending on the consistency (quantity and quality) of saliva, the difficulty of mineral entry and diffusion into saliva may pose a significant challenge to the bioavailability of minerals which come into contact with enamel surfaces and diffuse into the OB [20]. Despite saliva being in contact with the external portion of the OB, diffusion of ions and dissolved mineral species into the $\mathrm{OB}$ appears highly controlled [4,6]. It can be considered that this is because the $\mathrm{OB}$ contains micro fluid channels and exogenous proteins which can bind to and slow the entry and diffusion of various mineral species into the plaque fluid of the OB where acid attacks are prevalent $[4,19,20]$. This external portion commonly referred to as the exopolysaccharide slime layer or EPS controls the entry of ions into the plaque fluid of the OB which is the extracellular fluid in direct contact with tooth surfaces. Due to the isolated nature of the $\mathrm{OB}$, the ionic content of the plaque fluid which contacts tooth surfaces directly has its own separate gradient for dissolved mineral species which can further discourage free calcium bioavailability $[4,20]$.

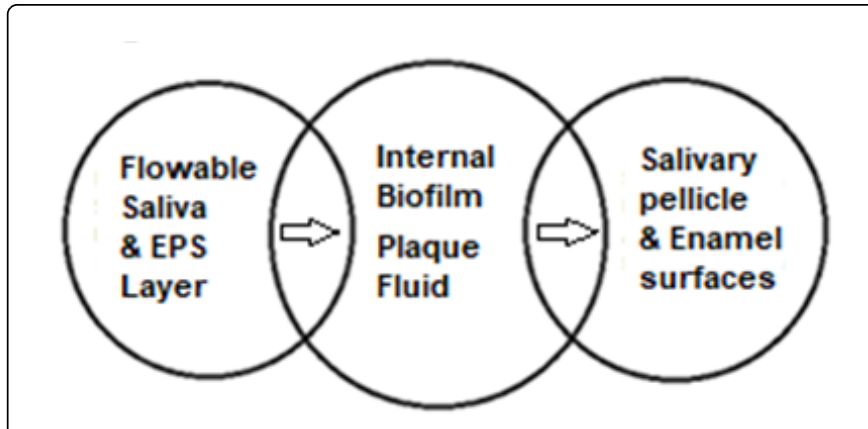

Figure 1(b): Ion gradients and barriers for saliva to neutralize plaque acids in the oral biofilm.

It has been hypothesized for some time that the plaque fluid of the $\mathrm{OB}$ is saturated with free calcium [4]. However, under acidic conditions $(\mathrm{pH}<5.5)$, the source of this free calcium is likely mainly derived from enamel and dentinal breakdown [11]. This is subsequently deleterious as the surface charge of the enamel under acidic conditions becomes more positive and can deter free calcium from favorably returning to promote remineralization [13]. This process is well understood and is known as Cation Exchange Capacity (CEC). When the CEC of a mineral surface increases, free calcium exchange, and deposition at HA surfaces also increases $[9,13]$. This is seen under more basic conditions $(\mathrm{pH}>7)$ and can be illustrated in Figure 1(a) with a stern based charge model [9]. As the $\mathrm{pH}$ Increases within the plaque fluid of the $\mathrm{OB}$, the surface charge of the enamel surfaces becomes more negative, allowing for higher free calcium exchange and integration onto the existing HA lattices $[9,13,21,22]$. This free calcium could also serve in a buffering capacity for future acid attacks [11]

Although many antibacterial agents have been highly relied upon to help aid against $\mathrm{OB}$ formation, the OB still remains a large problem. Even though topical antibacterial agents cannot remove or destroy a biofilm, they may be able to cause positive bacterial shifts back to less cariogenic bacteria by applying external pressure to biofilms during growth and colonization phases [23-25]. It is also possible that certain biofilm penetrants may increase the entry of other ions into the $\mathrm{OB}$ in a synergistic manner allowing increased exchange and calcium ion bioavailability within the plaque fluid of the OB. However, the usefulness of a biofilm penetrant largely depends on its required concentration for use, $\mathrm{pH}$, biocompatibility with human cells and potential side effects. In contrast to AgNPs, chlorhexidine gluconate has shown to potentially interfere with calcium and fluoride activity, especially at enamel surfaces: This makes it a less desirable choice for aiding in HA remineralization pathways [21].

By promoting shifts to bacteria which lack acidogenic potential on oral tissues, these material properties could yield conditions in which the $\mathrm{OB}$ could support improved calcium exchange onto tooth structures as well as keeping conditions within the biofilm less prone to cariogenic activity $[19,26]$. Biofilm penetrants which are small in size, do not impede calcium exchange, are stable under neutral to moderately basic conditions and are biocompatible with human tissues are ideal for this purpose.

Despite the well documented antibacterial properties of AgNPs, alternative properties of this interesting material have not been well explored [26]. It is the aim of this pilot study to evaluate whether or 
not AgNPs can be used as biofilm penetrants to directly increase free calcium activity within the $\mathrm{OB}$ under neutral to moderately basic conditions. If the use of AgNPs shows an increase in free calcium activity within the $\mathrm{OB}$ the possibilities for new synergistic remineralization pathways exist. This could also yield promising new alternatives and materials which are designed to reduce $\mathrm{OB}$ cariogenicity, and improve calcium bioavailability and exchange within the plaque fluid of the OB.

\section{Material and Methods}

\section{Study design}

This was a prospective, randomized, controlled, double-blinded case study. All participants provided written informed consent prior to study initiation.

\section{Subjects}

All subjects were patients of the practice (Mountain view dental). No recruitment methods were used. 5 male subjects and 4 female subjects were used for the first trial. For the second trial, 4 males and 4 females were used. Their ages ranged from 10-41 (mean age of 19).

\section{Inclusion criteria}

- All subjects have had a recent dental check-up and cleaning within the last 4-6 weeks

- Healthy hard and soft tissues based on a recent clinical exam

\section{Exclusion criteria}

- The recent history of caries (the Last check-up showed untreated caries)

- Presence of periodontal disease

- Any allergies to personal care, or cosmetic products

- Any medical condition requiring pre-medication prior to dental procedures

- Diminished immunity

- Reported poor wound healing or systemic diseases

- Hepatitis, HIV, ulcer forming diseases, abscesses, granulomas, or severe gingivitis or periodontitis

- Use of antibiotics within the last 3 months

- Tobacco use, smoking

- Pregnant or lactating

\section{Clinical case protocol}

In order to test the hypothesis that biofilm penetrants can be used to increase the bioavailability and surface exchange of calcium ions within the $\mathrm{OB}$, an accepted procedural method for testing the biofilm plaque fluids was needed. This method was replicated from previous studies done by Vogel et al. This method has been shown to be effective for analyzing ionic activities of various ions within the plaque biofilm and is highly replicable. Activity is the measure of "active" free ions in solution and is used to determine how much bio-available free calcium is present in the plaque fluid of the $\mathrm{OB}$ for reactions with tooth surfaces. The higher the activity measured, the more free calcium is present. All subjects provided written informed consent and agreed to be a part of the case study prior to initiation. All subjects abstained from brushing and flossing 48 hours prior to the case study and fasted the night before this study. Two plaque samples were taken for each patient using a plastic instrument. The two samples were taken including a baseline sample and a sample taken 60 minutes after rinsing. Two trials were performed. The first trial tested each individual's pre and post-rinse samples, and shows averaged data. The second trial tested pre and post-rinse pooled samples from multiple individuals. It can be noted that due to limitations of current microelectrodes, that individual plaque samples pose much more difficult to produce stable readings, and thus are more prone to error. This is largely due to the fact that the sample sizes are so small, that subsequent low plaque fluid volumes were difficult to test. It can be recommended that larger pooled sample sizes are far more reliable and produce a very low error in comparison as seen in trial \#2.

In this case study, two rinses were made. Rinse A was a control rinse and contained $0.2 \%$ of Calcium Chloride, and rinse $\mathrm{B}$ was an experimental rinse and contained $0.2 \%$ of Calcium Chloride in addition to $10 \mathrm{ppm}$ of Silver Nanoparticles (average diameter of $<10$ $\mathrm{nm}>$ capped with a proprietary polysaccharide). Both rinses were tested at a $\mathrm{pH}$ of 7.2. The Nanoparticles were provided from the Center for Advanced materials processing from Clarkson University, NY, and diluted down for the purpose of the study.

Both rinses were made from the same base solution and verified to have the same $\mathrm{mV}$ reading upon insertion of the Calcium electrode in order to show that AgNPs at the specified size, $\mathrm{pH}$ and concentration used did not result in any interference of results from the readings taken. All subjects were randomly assigned to a rinse and asked to rinse for 60 seconds after a baseline sample was taken. All participants chosen were caries free. Two trials were done. Only one practitioner had access to the sample lots. Otherwise, all investigators and subjects were blinded with regard to the mouth rinse sample application. Subjects only had to come for one visit for the case study and were told not to use any hygiene measures for 48 hours prior including rinsing, brushing, flossing and chewing gum. All participants were sent home with instructions on what do and abstained from hygiene prior to testing. At the visit, and 24 hours after, all subjects were questioned about adverse effects of any products. A 24-hour emergency line was provided to all subjects in case of emergency. No adverse events were reported.

Trial\# 1 was done with nine qualifying caries-free individuals whose before and after samples were centrifuged and analyzed separately. Trial \#2 was done with 8 qualifying caries-free individuals whose initial and post-rinse plaque samples were pooled into four microcentrifuge tubes. This case study was modeled off of Vogel et al., whereby plaque samples were collected and immediately placed under mineral oil, centrifuged at 14,000 RPM for 15 minutes at 5 degrees $\mathrm{C}$ and tested shortly after. A Microelectrode (KWIK-CAL2) and subsequent reference electrode from World Precision instruments were used to test samples directly for the activity of calcium $\left(\mathrm{Ca}^{2+}\right)$. Calibrations of the electrode were done in accordance with the manufacturer's recommendations and all samples were analyzed immediately and kept at the same temperature ( 5 degrees Celsius) to avoid error. After analysis, samples were disposed of.

\section{Data Analysis}

The difference between endpoint and baseline data values was determined for each participant using a 2 -sided T-test for significance. 
The calcium values obtained from electrodes used extra orally were taken for the test and control rinses.

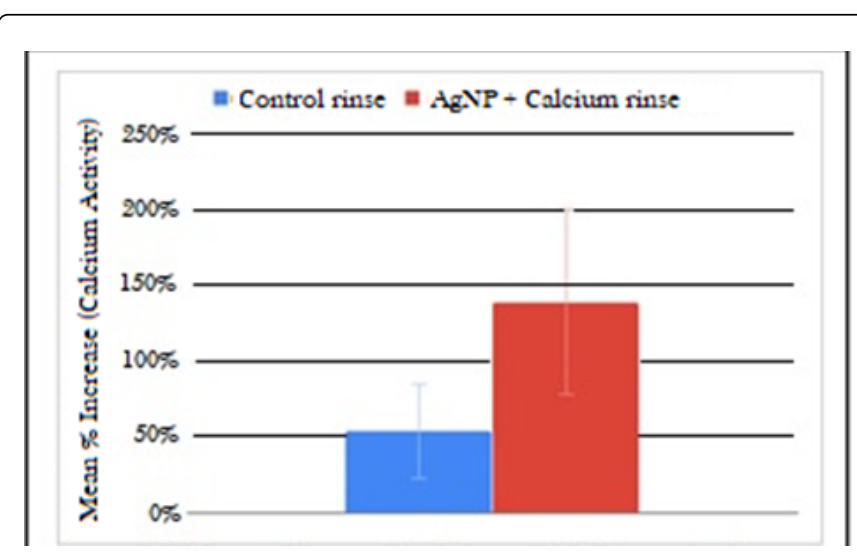

Figure 2(a): Mean\% increase in calcium activity (average of individual plaque samples): control rinse vs nanosilver+calcium test rinse

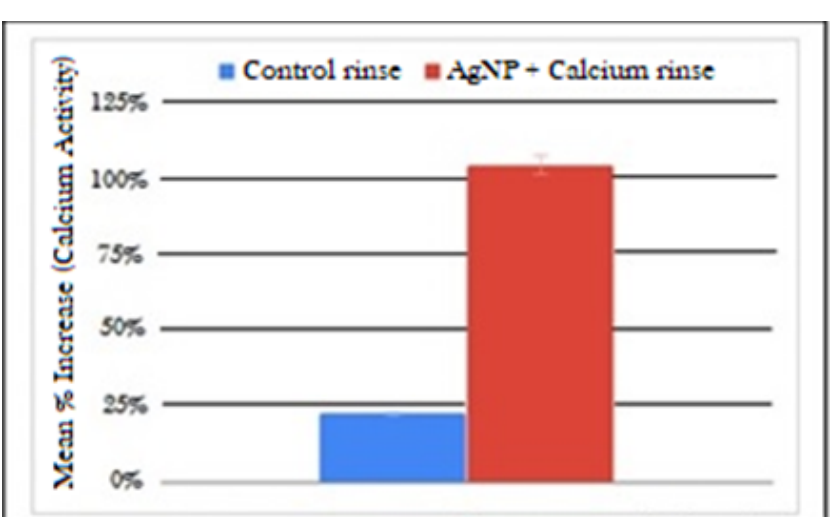

Figure 2(b): Mean\% increase in calcium activity (pooled plaque samples): control rinse vs nanosilver+calcium test rinse.

\section{Results}

The two trials primary differences revolved around plaque sample sizes. In previous studies, it has been noted that due to the limitations with micro-electrodes and small fluid volumes from plaque samples, that pooling samples is a more effective method of obtaining more precise and accurate results with less error. All samples taken were tested individually (Trial \#1) and in a pooled format (Trial \#2). All readings were taken in triplicate. It was noted that although results in Trial \#1 showed $(\mathrm{p}<0.10)$, that the margin of error in Trial \#2 was far lower and statistically significant $(p<0.05)$ due to the consistency and stability of the microelectrode readings from larger sample sizes. Both trials showed important findings, but for purposes of maintaining the highest significance, the discussion will focus on trial \#2 which had pooled plaque samples.

As can be noted in Figure 2(a) and 2(b), the results from the experiment showed significant increases in free calcium activity in the plaque fluid of the plaque biofilm. The experimental rinse (Rinse B) showed a 4.6 times increase in free calcium activity $(\mathrm{p}<0.05)$ when compared to the increase from the control (Rinse A), which contained the same amount of calcium chloride but did not have silver nanoparticles. It can be hypothesized that this large increase is due to the breakdown of micro-channels in the EPS slime layer which the OB utilizes to control the net ionic flow of ions.

\section{Discussion}

As can be noted from the results, it is clear that AgNPs are a very promising material which can potentiate the bioactivity and exchange of Calcium Ions in the plaque fluid of the OB. It is warranted that a larger scale study should be done in order to verify the extent of its effects on calcium activity within the OB. It can be noted that increased formulary optimization could also occur if the form of calcium salt is changed, which was not analyzed in this case study. As different salts should be trialed, it is important that the combination of silver nanoparticles and calcium salts do not cause interference or instability with one another, especially over long periods.

It can be noted that forms of calcium that produce moderately basic solutions ( $\mathrm{pH}=7.0-8.0$ ) would be ideal for remineralization. It has been noted that the synthesized nanoparticles were highly salt stable, and agglomeration was not observed during the experiment. In the future, highly stable Silver nanoparticles which remain stable in the presence of high salt, osmolyte concentrations, and in a wide $\mathrm{pH}$ range could be extremely useful for dental applications, as their increased stability could result in more predictable use for long term delivery and use for hygiene applications.

Under conditions of a stable moderately basic solution with low to moderate salt ratios, further increases in calcium ion bioavailability and remineralization potential could be achieved. This can significantly alter the ability of the $\mathrm{OB}$ over time to undergo negative shifting and can prevent the ultimate release and subsequent neutralization of acidic constituents that damage tooth structures. Promotion of an increased $\mathrm{pH}$, alongside a higher bioavailability of calcium ions with the $\mathrm{OB}$, can lead to the substantial potential for remineralization through a high CEC and low interference from acidic ions for surface sites $[13,18]$.

\section{Conclusion}

With the advent of new technological advances, material sciences have expanded dramatically. Silver nanoparticles have seen wide applications throughout industry and will serve as a key starting point for potential use against oral biofilms [26]. While oral hygiene regimens have remained the same for some time, the $\mathrm{OB}$ continues to evolve and develop ways to combat the natural defense mechanisms the body utilizes to prevent decay.

Although this is a pilot study, which will need to be repeated on a larger scale to make systemic conclusions, the concept that AgNPs can be utilized as a biofilm penetrant to alter the gradients of the external $\mathrm{OB}$ and allow larger influxes of calcium ions proves to be a very relevant finding for potential use. AgNPs are not only larger than the calcium ions but are not as attracted to Enamel surface sites due to their like charge at neutral to basic conditions. Compared to chlorhexidine, which has been shown to bind to enamel surfaces and potentially deter calcium and fluoride absorption [21], AgNPs have the potential to promote a non-competitive environment for free calcium ions to deposit onto enamel surface sites [11]. Such a combination should be studied more extensively and has been shown in this pilot study to drastically improve the activity of free calcium ions within the 
plaque fluid of the plaque biofilm. Although it has been shown in previous studies that small silver nanoparticles $(<2.7 \mathrm{~nm}>)$ can be utilized in composite and adhesive applications with calcium agents to offer the dual additive benefits from each, it has never before been shown that AgNPs have synergistic activity on the oral biofilm's uptake of free calcium into plaque fluid [24]. This has direct relevance since plaque fluid is in constant contact with tooth structures and does not always reflect the $\mathrm{pH}$ and ionic composition of saliva [11]. AgNPs in combination with a stabilized calcium salt shows great synergistic potential to reduce decay risk and subsequent risk factors associated with the development of biofilm carcinogenicity in plaque fluid. It is recommended this mechanism be further studied and explored to verify the extent of this synergistic behavior.

\section{Authors Contributions}

All Authors contributed to concept/study design, data collection and article drafting.

\section{Conflict of Interest}

All Authors have filed patent applications surrounding the research applications, all Authors acknowledge interest in ownership of Novus Research LLC.

\section{Acknowledgement}

Thanks go to the team at Mountain View Dental Group who let us use their facility for collection of samples and equipment analysis.

\section{References}

1. Saini R, Saini S, Sharma S (2011) Biofilm: A dental microbial infection. J Nat Sci Biol Med 2: 71-75.

2. Uzel NG, Teles FR, Teles RP, Song XQ, Torresyap G, et al. (2011) Microbial shifts during dental biofilm re-development in the absence of oral hygiene in periodontal health and disease. J Clin Periodontol 38: 612-620.

3. Masadeh MM, Gharaibeh SF, Alzoubi KH, Al-Azzam SI, Obeidat WM (2013) Antimicrobial activity of common mouthwash solutions on multidrug-resistance bacterial biofilms. J Clin Med Res 5: 389-394.

4. Margolis HC, Moreno EC (1994) Composition and cariogenic potential of dental plaque fluid. Crit Rev Oral Biol Med 5: 1-25.

5. Ready D, Bedi R, Spratt DA, Mullany P, Wilson M (2003) Prevalence, proportions, and identities of antibiotic-resistant bacteria in the oral microflora of healthy children. Microb Drug Resist 9: 367-372.

6. Margolis HC, Moreno EC (1992) Composition of pooled plaque fluid from caries-free and caries-positive individuals following sucrose exposure. J Dent Res 71: 1776-1784.

7. Takagi S, Liao H, Chow LC (2000) Effect of tooth-bound fluoride on enamel demineralization/remineralization in vitro. Caries Res 34: 281-288.
8. Schoonheydt RA, Johnston CT (2006) Surface and interface chemistry of clay minerals. 1: 87-113.

9. The Society for Sedimentary Geology (SEPM) Clay minerals for petroleum geologists and engineers. Copyright, (SC22), 1988.

10. Bell LC, Posner AM, Quirk JP (1973) The point of zero charge of hydroxyapatite and fluorapatite in aqueous solutions. J Colloid Interface Sci 42: 250-261.

11. Dawes C (2003) What is the critical pH and why does a tooth dissolve in acid? J Can Dent Assoc 69: 722-724.

12. Arends J, Jongebloed WL (1977) The enamel substrate-characteristics of the enamel surface. Swed Dent J 1: 215-224.

13. Eslinger E, Pevear DR (1988) Clay minerals for petroleum geologists and engineers. SEPM.

14. Shawky HA, Basha SM, Batouti GA, Kassem AA (2015) Evaluation of clinical and antimicrobial efficacy of silver nanoparticles and tetracycline films in the treatment of periodontal pockets. IOSR J Dent Med Sci Ver I 14: 2279-2861.

15. Leitão TJ, Cury JA, Tenuta LMA (2018) Kinetics of calcium binding to dental biofilm bacteria. Das S, ed. PLoS One 13: e0191284.

16. Featherstone JDB (2008) Dental caries: a dynamic disease process. Aust Dent J 53: 286-291.

17. Dai L, Liu Y, Salameh Z (2010) Can caries-affected dentin be completely remineralized by guided tissue remineralization? Dent Hypotheses 1: 59-68.

18. Singh ML, Papas AS (2009) Long-term clinical observation of dental caries in salivary hypofunction patients using a supersaturated calciumphosphate remineralizing rinse. J Clin Dent 20: 87-92.

19. Astasov-Frauenhoffer $M$, Varenganayil MM, Decho AW, Waltimo $T$, Braissant O (2017) Exopolysaccharides regulate calcium flow in cariogenic biofilms. PLoS One 12: e0186256.

20. Amaral COF do, Straioto FG, Napimoga MH, Martinez EF (2017) Caries experience and salivary aspects in individuals with fragile $\mathrm{X}$ syndrome. Braz Oral Res 31: e79.

21. Autio-Gold J (2008) The role of chlorhexidine in caries prevention. Oper Dent 33: 710-716.

22. Sekine Y, Motokawa R, Kozai N, Ohnuki T, Matsumura D, et al. (2017) Calcium-deficient Hydroxyapatite as a Potential Sorbent for Strontium. Sci Rep 7: 2064.

23. Marsh PD (2006) Dental plaque as a biofilm and a microbial communityimplications for health and disease. BMC Oral Health 6: S14.

24. Zhang K, Wang S, Zhou X, Xu HHK, Weir MD, et al. (2015) Effect of antibacterial dental adhesive on multispecies biofilms formation. J Dent Res 94: 622-629.

25. Latimer J, Munday JL, Buzza KM, Forbes S, Sreenivasan PK, et al. (2015) Antibacterial and anti-biofilm activity of mouthrinses containing cetylpyridinium chloride and sodium fluoride. BMC Microbiol 15: 169.

26. Noronha VT, Paula AJ, Durán G, Galembeck A, Cogo-Müller K, et al. (2017) Silver nanoparticles in dentistry. Dent Mater 33: 1110-1126. 